\title{
Parametric instabilities in 3D periodically focused beams with space charge
}

\author{
Ingo Hofmann \\ Technische Universität Darmstadt, Schlossgartenstrasse 8, 64289 Darmstadt, Germany; \\ GSI Helmholtzzentrum für Schwerionenforschung GmbH, Planckstrasse 1, 64291 Darmstadt, Germany \\ and Goethe Universität, Max-von-Laue-Strasse 1, 60438 Frankfurt am Main, Germany
}

Oliver Boine-Frankenheim

Technische Universität Darmstadt, Schlossgartenstrasse 8, 64289 Darmstadt, Germany and GSI Helmholtzzentrum für Schwerionenforschung GmbH, Planckstrasse 1, 64291 Darmstadt, Germany (Received 26 October 2016; published 11 January 2017)

\begin{abstract}
Parametric resonances of beam eigenmodes with a periodic focusing system under the effect of space charge-also called structural instabilities - are the collective counterparts to parametric resonances of single particles or of mechanical systems. Their common feature is that an exponential instability is driven by a temporal modulation of a system parameter. Thus, they are complementary to the more commonly considered space charge single particle resonances, where space charge pseudo-multipole terms are assumed to exist already at finite level in the initial distribution. This article elaborates on the characteristics of such parametric instabilities in 3D bunched beams-as typical in linear accelerators-for modes of second (envelope), third and fourth order, including the transverse coupled "sum envelope instabilities" recently discovered for 2D beams. Noteworthy results are the finding that parametric resonances can be in competition with single particle resonances of twice the order due to overlapping stopbands; furthermore the surprisingly good applicability of the stopband positions and widths obtained from previously published 2D linearised Vlasov stability theory to the 3D non-Kapchinskij-Vladimirskij particle-in-cell code studies presented here.
\end{abstract}

DOI: 10.1103/PhysRevAccelBeams.20.014202

\section{INTRODUCTION}

An understanding of space charge effects in beam dynamics of both linear and circular high intensity accelerators is crucial for the design, operation, and optimization of such accelerators. The most frequently considered space charge effect is its impact on the tune spread for resonances caused by magnetic field nonlinearities or magnet errors. Here we consider, instead, the possibility that space charge itself provides a resonant driving force in an otherwise strictly linear periodic focusing structure. Generally speaking, one needs to distinguish between two kinds of space charge induced resonances: (i) the "single particle resonance," where the initial density profile-assuming that it is nonuniform-already provides a space charge "pseudomultipole" as a resonance driving term; (ii) the parametric resonance or instability, where a system parameter is periodically modulated and drives an eigenmode of the beam, which may have existed only on the noise level

\footnotetext{
*i.hofmann@gsi.de

Published by the American Physical Society under the terms of the Creative Commons Attribution 4.0 International license. Further distribution of this work must maintain attribution to the author(s) and the published article's title, journal citation, and DOI.
}

initially-as is the case with the well-known envelope instability in a stop-band near $90^{\circ}$ phase advance.

The focus of this paper is on the second case. A useful theoretical basis and guidance for parametric instabilities in intense beams was laid in the 2D (coasting beam) Vlasov equation perturbation analysis of a KapchinskijVladimirskij (KV) distribution in periodic focusing of Ref. [1]. This work included the envelope instability occurring at $90^{\circ}$ phase advance and demonstrated that similar parametric instability phenomena also exist in higher order-even suggesting up to arbitrarily high order for an initial KV-distribution. The envelope instability was studied theoretically in further detail in Ref. [2], and more recently in Ref. [3] as well as in Ref. [4]. Not surprisingly, in realistic non-KV beams most of the high order instabilities are absent or negligible. However, questions in connection with 3D beams - in particular short ellipsoidal bunches as in linear accelerators - and with unequal tunes in $x, y$, or $z$ have not been addressed systematically up to now. For clarification we emphasize that the notation parametric instability is not commonly used in literature on the envelope instability. We find it adequate to emphasize the parallelism with other disciplines, where analogous phenomena exist, including the sum parametric instabilities.

On the experimental side only limited data exist in spite of an early observation of the phenomenon in periodic 
transport of electron beams by Pierce [5]. The first detailed experiment using ions was with a coasting beam in a periodic channel by Tiefenback [6,7] to test the theory of Ref. [1]. It confirmed significant beam degradation above $90^{\circ}$ phase advance and remained the primary experimental evidence for over three decades. In a linac environment the first test of the $90^{\circ}$ stopband for bunched beams was undertaken in 2009 in the UNILAC high intensity heavy ion accelerator [8]. This experiment left open questions of interpretation, which were addressed in recent theoretical research clarifying the joint appearance of second order parametric (envelope instability) and fourth order single particle resonant space charge effects [9]. Additional studies followed indicating that the subject remains of interest, but also that full agreement between different authors on the interpretation of the phenomena is still pending $[10,11]$.

The present study is an attempt to present these parametric phenomena in 3D bunches in a more systematic way from second to fourth order-including the recently discovered 2D "sum parametric effects" [12]. We proceed with a short review of the analytical description of parametric resonances (KV-instabilities) in periodic focusing in Sec. II; continue with an application to the $90^{\circ}$ stopband in Sec. III; present an overview of parametric effects in second order including sum resonances in Sec. IV; discuss higher order parametric effects in Sec. V, present an overview stability diagram in Sec. VI and offer an outlook in Sec. VII.

Note that all examples in the simulation part of our study are related to short bunches, with longitudinal oscillation periods close to betatron periods as typical for linear accelerators; in circular accelerators the synchrotron period is usually significantly longer, which requires reconsideration of our findings due to possibly significant effects caused by Landau damping. For simplicity we also assume equal emittances in all planes and leave out emittance exchange effects, which are not driven parametrically, but by anisotropy [13].

\section{REVIEW OF PARAMETRIC RESONANCES IN PERIODIC FOCUSING}

\section{A. Notations for parametric resonance}

The most common case of parametric resonance in accelerators is the single-particle "Mathieu stopband". It has the effect to prevent particle transport in periodic focusing, if the phase advance is $180^{\circ}$ per focusing period. This follows the well-known behavior of a linear differential equation of Hill's type, $d^{2} x / d t^{2}+\left[\omega^{2}+f(t)\right] x=0$. Here $\omega$ is the eigenfrequency of the free oscillation and $f(t)$ is assumed to be a parameter of the oscillating system, which varies periodically with $\omega_{0}$. Exponential instability of an arbitrarily small initial perturbation results, if

$$
\omega=\frac{n}{2} \omega_{0} .
$$

Here $n$ is a positive integer expressing the parametric order. The most pronounced case of this parametric resonance-with the widest stopband-is the "halfinteger" case, $n=1$, often called "parametric instability" or "sub-harmonic instability" [14], with the envelope instability as an example.

In parametric resonance theory involving several coupled equations so-called "combination" resonances are known to exist $[14,15]$, where two or more eigenmodes of the system jointly enable a parametric resonance via a sum resonance condition $\omega_{1}+\omega_{2}=\omega_{0}$. Recently, such "sum parametric resonances" between two envelope equations in 2D with coupling between two eigenmodes were found [12], which will be applied here to transverse coupling in ellipsoidal bunches.

Note that in the following $\omega$ relates to coherent eigenmodes of the beams, rather than to single particle frequencies. The phase advances of particles-their rms values-are written in linac notation as degrees per focusing cell: $k_{0, x, y, z}$ without space charge, and $k_{x, y, z}$ with space charge. Applied to circular accelerators, these quantities would have to relate to a periodic structure cell.

Using linac notation the parametric resonance condition Eq. (1) for an eigenmode in the $x-x^{\prime}$-plane can be written in degrees as:

$$
\phi \equiv m k_{0, x}-\Delta k_{m, \mathrm{coh}}=\frac{n}{2} 360^{\circ},
$$

where $m$ relates to the order of the resonance in the $x-x^{\prime}$-plane and $\Delta k_{m, \text { coh }}$ (generally $>0$ ) stands for a coherent space charge tune shift (in the present notation it also includes the incoherent tune shift for simplicity). The latter is proportional to the single particle tune shift and depends on the specific eigenmode, hence on $m, n$ and possibly other parameters. In practice, $\Delta k_{m, \mathrm{coh}}$ is a measure for the shift of the center of stopband due to space charge. For smooth approximation and KV-beams an overview on coherent space charge tune shifts, with a distinction between "even" and "odd" (skewed) modes, is presented in Ref. [16]).

In the notation of circular accelerators the equivalent condition is written as:

$$
\phi \equiv m Q_{0, x}-\Delta Q_{m, \mathrm{coh}}=\frac{n}{2} N,
$$

where $N$ relates to the number of structure cells (superperiods) per circumference and $\Delta Q_{m \text {,coh }}$ is again a coherent tune shift.

\section{B. Envelope instability}

As an example of a half-integer parametric resonance we consider the transverse envelope instability of an ellipsoidal bunch in a symmetric periodic focusing-drift-defocusingdrift array of quadrupoles, with longitudinal focusing 
provided by two thin rf gaps in the center of both drift spaces in each cell. We use the 3D KV-envelope equation option of the TRACEWIN code [17], a parameter setting within a stopband of instability assuming $k_{0, x, y}=100^{\circ}$, a moderate space charge leading to $k_{x, y}=82^{\circ}$, and the longitudinal focusing arbitrarily set to $k_{0, z}=50^{\circ}$.

Results for the parametric instability case according to $\phi=2 k_{0, x, y}-\Delta k_{2, \text { coh }}=180^{\circ}$ are shown in Fig. 1. The initial exponential phase is followed by phases of saturation of growth, damping, and again growth with a chaotic exchange between $x$ and $y$. Details of the lattice and envelope periods are shown in the insert. The three rms emittances are initially chosen equal, which results in bunches slightly elongated from spherical. It should also be noted that in the envelope model coupling between different degrees of freedom is by means of the associated space charge force modulation, which is a weak but not unimportant effect.

The full response curve employing the envelope maxima in a 500 cell long lattice is shown in Fig. 2 for the case of $k_{0, x, y}=100^{\circ}$ and variable $k_{x, y}$. Note the sharp maximum at the right edge, with a steep drop to zero, which is a strong coherent effect. Entering with $k_{x, y}$ into the stopband at the right edge has a strongly "attractive" effect: an infinitesimal dilution of space charge causes a corresponding increase of $k_{x, y}$ with more growth. This continues self-consistently, until $k_{x, y}$ reaches the left edge of the-also dynamically evolving - stopband. Entering at the left edge, instead, pushes $k_{x, y}$ backwards and stops growth. An interesting connection between emittance growth and the saturation time needed for crossing the stopband is discussed in Ref. [4].

A necessary criterion for instability is $k_{0, x, y}>90^{\circ}$ with at the same $k_{x, y}$ below $90^{\circ}$. This enclosure of $90^{\circ}$ justifies the nomenclature $90^{\circ}$-stopband.

\section{KV-instabilities}

In a uniform density KV-beam propagated in a linear, periodic focusing lattice space charge forces are also linear, hence emittances remain invariant in the absence of acceleration. Treating the envelope instability with the $\mathrm{KV}$-envelope equations as discussed in Sec. II B leads to
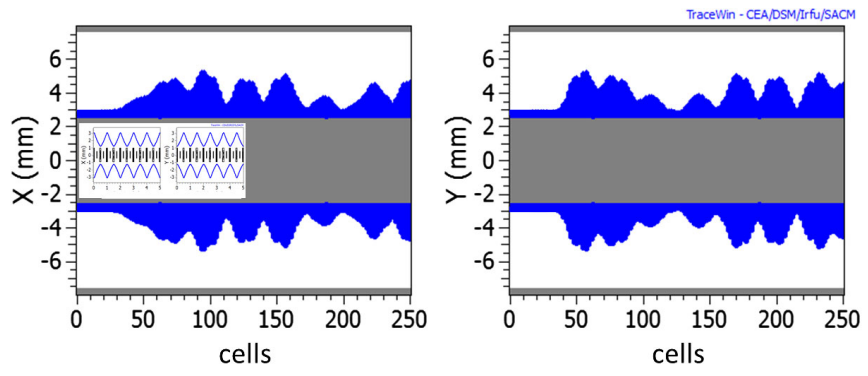

FIG. 1. Evolution of KV-envelopes versus cell number for $k_{0, x, y}=100^{\circ}, k_{x, y}=82^{\circ}$.

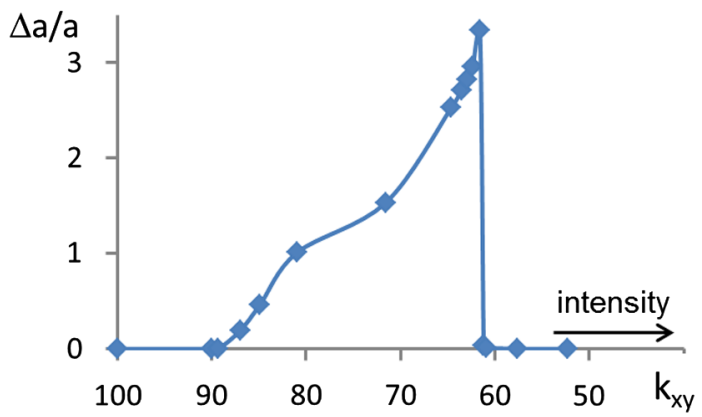

FIG. 2. Complete stopband with relative growth of rms envelopes for fixed $k_{0 x, y}=100^{\circ}$ as function of $k_{x, y}$.

mismatch, but no rms emittance change or density nonuniformity. This requires higher than second order parametric resonances, or instabilities, which have the effect that different orders of space charge "pseudo-multipoles" get parametrically driven from noise to a finite level.

Although 2D KV-distributions are not a choice for realistic beam simulations, they are the only ones that allow analytical calculation of the associated parametric resonance stopbands, which was the motivation behind the early theoretical work in Ref. [1]. Its goal was to explore high current limits for very long bunches in the context of the heavy ion inertial fusion ideas of the 1970s — at a time when computer simulation of space charge dominated beams was just at its beginning. In fact, we find that for low order $m$ they provide quite a useful basis for stopband widths even for 3D short bunches and realistic distributions.

In Fig. 3 we start with a short review of results from Ref. [1], which apply to symmetric periodic focusing and to equal tunes and emittances in both degrees of freedom. By carrying out a perturbation analysis of the linearized Vlasov and Poisson equations, second and higher order moments are obtained, and their stability is checked. Stopbands are defined as regions where nonzero exponential growth rates are found.

All envelope instability stopbands require $k_{0, x, y}>90^{\circ}$ and set in for $k_{x, y}$ slightly under $90^{\circ}$. This reflects the effect of $\Delta k_{2, \text { coh }}$, which includes also the incoherent tune shift. The unstable envelope behavior of Fig. 1 is confirmed, but comparing the curve for $k_{0, x, y}=100^{\circ}$ with Fig. 2, it is noted that at high intensity the latter is more extended by $10^{\circ}$; this difference may be a result of comparing 2D coasting beams with 3D ellipsoidal bunches.

Results for third and fourth order parametric resonances are shown in the center, respectively bottom graphs of Fig. 3. The third order mode stopband requires $k_{0, x, y}>60^{\circ}$ and sets in for $k_{x, y}$ under $60^{\circ}$. Its width shrinks to zero for $k_{0, x, y} \rightarrow 60^{\circ}$ (not shown here). The resonance is of the kind $\phi=3 k_{0, x, y}-\Delta k_{3, \mathrm{coh}}=\frac{1}{2} 360^{\circ}$, hence a (half-integer) parametric instability.

In fourth order, with $\phi=4 k_{0, x, y}-\Delta k_{4, \mathrm{coh}}=\frac{n}{2} 360^{\circ}$, a larger number of stopbands exists, but only even modes 

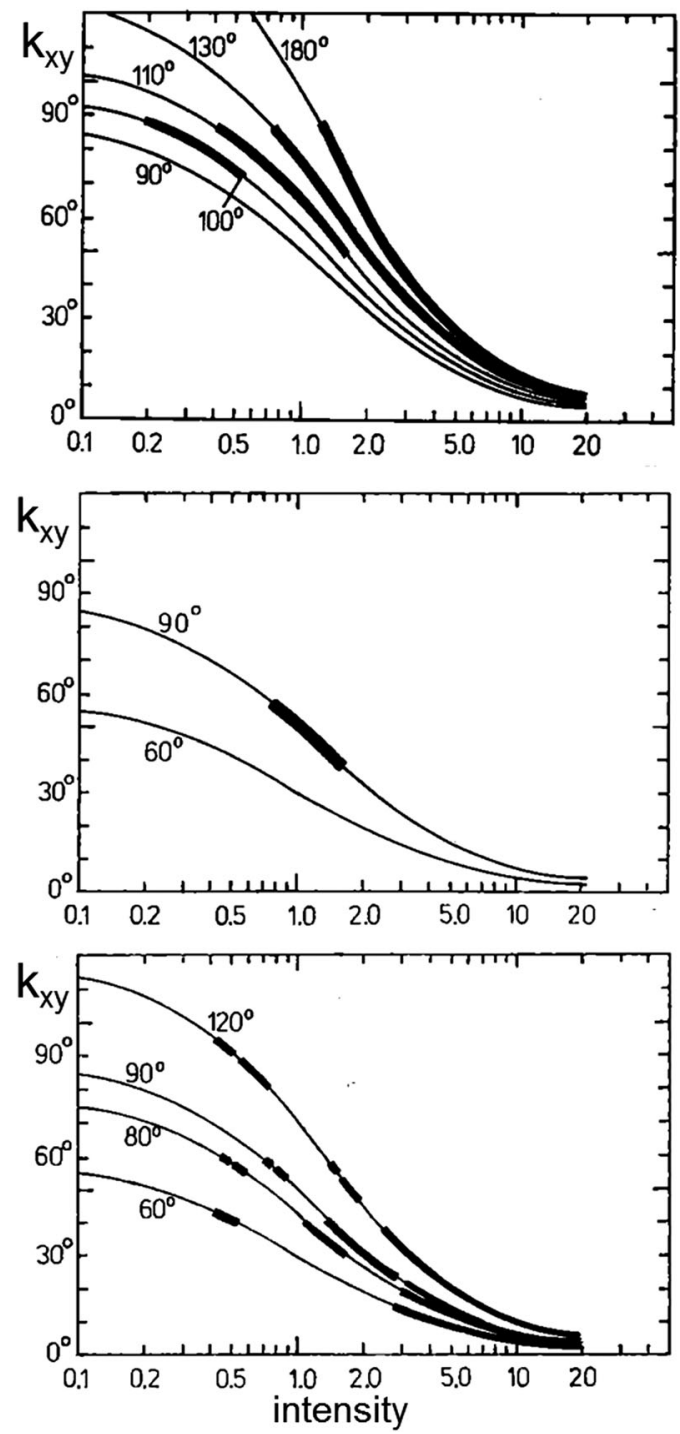

FIG. 3. Stopbands of second order (top, envelope instability), third order (center) and fourth order (bottom) parametric resonances as function of a dimensionless intensity parameter. Each curve relates intensity to $k_{x, y}$ for a fixed value of $k_{0, x, y}$ (in degrees) (source: Ref. [1]).

are displayed in Fig. 3. For $k_{0, x, y}=120^{\circ}$ we recognize a pair of nearly adjacent stopbands for $k_{x, y}$ slightly under $90^{\circ}$, which can be assumed to shrink in width and merge to the value $k_{0, x, y}=90^{\circ}$ for vanishing intensity. Different from the above envelope and third order examples, this $n=2$ mode obeys an integer parametric relationship $\phi=4 k_{0, x, y}-\Delta k_{4, \mathrm{coh}}=360^{\circ}$. However, also a fourth order mode with half-integer relationship case $\phi=4 k_{0, x, y}-$ $\Delta k_{4, \mathrm{coh}}=\frac{1}{2} 360^{\circ}$ exists for $k_{0, x, y}>45^{\circ}$, if $k_{x, y}$ is slightly under $45^{\circ}$. The associated patch of instability merges into $k_{0, x, y}=45^{\circ}$ for vanishing intensity.

We summarize this section by observing that due to the linearized KV-model behind the diagrams of Fig. 3 some caution is required in applying them to more general distributions. Only part of the instabilities-primarily the above listed modes-are retrieved in simulations and applicable to waterbag distribution functions.

Note that the extended stopbands for the intensity parameter exceeding " 3 ," for example, are not parametric and a KV-artefact (discussed as "Gluckstern-modes" in the literature); they have no relevance for realistic — sufficiently broadened - distribution functions like waterbag, which is discussed in more detail in Ref. [18].

\section{THE 90 ${ }^{\circ}$ PARAMETRIC STOPBAND}

The behavior of beams at $90^{\circ}$ phase advance-assumed as upper limit for focusing in linac design-has been the subject of different interpretations about the actual mechanism [8,9,11]. According to Ref. [9], the stopband is a coincidence of an envelope instability and a fourth order resonance, where the balance depends on the tune value and other factors. Comparison with the location of stopbands in Fig. 3 helps clarifying this interplay of the two modes.

We use the TRACEWIN code particle-in-cell option to model a short bunched beam with identical parameters as shown in Fig. 1. The bunch is longitudinally contained in a rf bucket and has only a few degrees of phase width to ensure linear longitudinal if forces. The synchronous phase is $-90^{\circ}$, hence no acceleration. Thus changing tunes are avoided, and results remain general and independent of a specific scheme of acceleration. The number of simulation particles is chosen as 128.000 , which yields a sufficiently low noise level for a grid parameter $n_{c}=8$ [19]. The selfconsistent treatment of the bunch space charge potential allows studying the evolution of all kinds of higher order terms in the space charge potential.

We start with an example, where $k_{0, x, y}=120^{\circ}$ and initially $k_{x, y}=80^{\circ}$-located inside but close to the lower edge of the stopband of the even mode fourth order parametric resonance in Fig. 3. As the first example in the top graph of Fig. 4 we employ the TRACEWIN option for a transverse KV-distribution beam and uniform (random) filling of the longitudinal phase plane ellipse, which is locally (e.g., along the bunch) equivalent to a coasting beam 2D KV-distribution. The transverse rms emittances of the KV case increase quickly by about $80 \%-90 \%$ in the average, with unambiguous indication of a fourth order resonance in both planes (see four-fold phase space symmetry shown at cells 8 and 18 , which get only smeared out at later times). Note that the initial distribution has strictly uniform transverse density profiles with no space charge "pseudo-octupole" content, except on the noise level. Thus, this growth can be attributed only to an integer $(n=2)$ parametric resonance $\phi=4 k_{0, x, y}-\Delta k_{4, \mathrm{coh}}=360^{\circ}$, which drives its amplitude to a finite level until saturation. The existence of this mode is in agreement with the linearized theory stopband prediction at $90^{\circ}$ of Fig. 3 .

For the initial waterbag (bottom graph of Fig. 4) the rms emittance growth result is surprisingly similar, but with a 

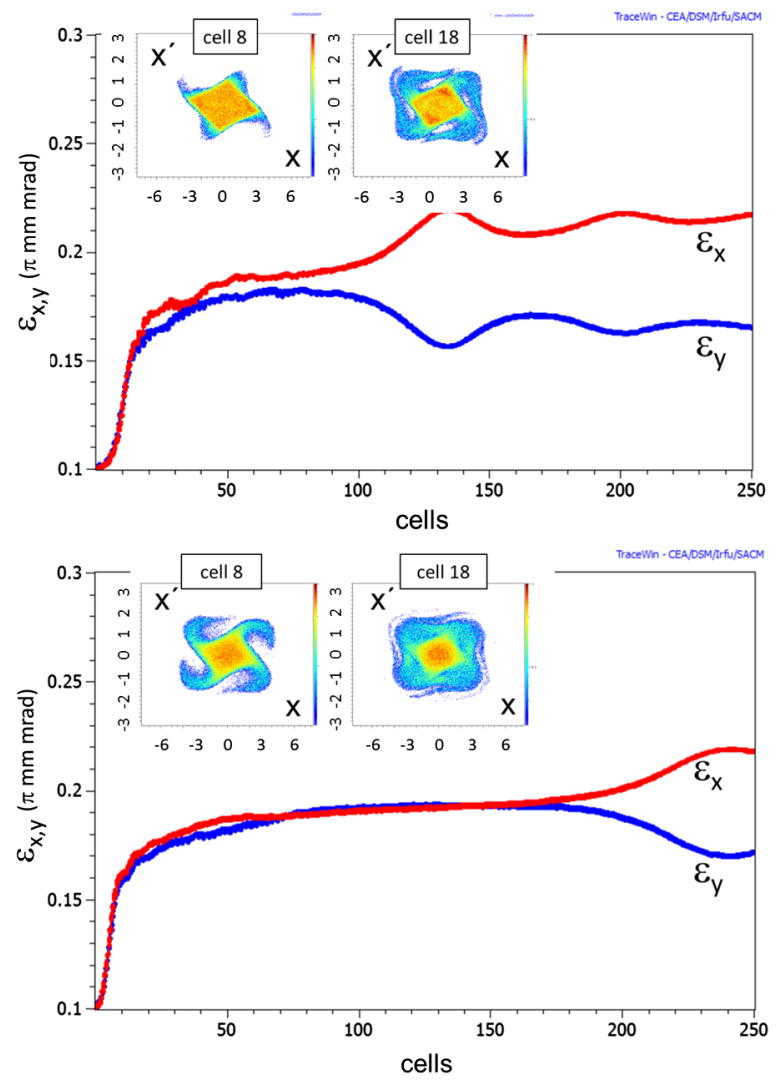

FIG. 4. rms emittances versus cell number for $k_{0, x, y}=120^{\circ}$, $k_{x, y}=80^{\circ}$ (inserts showing phase space plots at indicated cells) for $\mathrm{KV}$ beam (top graph) and for waterbag distribution beam (bottom graph).

steeper initial rise. A pronounced four-fold phase space plot is already evident at cell 8 . We conclude that here the presence of a finite space charge "pseudo-octupole" in the initial density profile results in an immediate "single particle" (incoherent) resonance action following $4 k_{x, y} \equiv 4\left(k_{0, x, y}-\Delta k_{\text {inc }}\right)=360^{\circ}$.

It is interesting to observe the time evolution of the rms phase advance during the phase of emittance growth, which results in a self-consistently increasing $k_{x, y}$ as discussed in Sec. II B. The saturated value reached near cell 50 in Fig. 5 indicates a $k_{x, y} \approx 97^{\circ}$, which agrees well with the upper-low space charge density-edge of the fourth order mode stop-band in Fig. 3. This is in spite of the fact that the latter is based on an instability of the 2D KV-distribution, while the simulation uses a $6 \mathrm{~d}$ waterbag distribution with an initial and not insignificant space charge "pseudo-octupole."

For both, KV and waterbag distribution, no indication of envelope instability is found in this case. However, with a slight increase of the starting intensity to reach the value $k_{x, y}=73^{\circ}$ (keeping $k_{0, x, y}=120^{\circ}$ ), we find in Fig. 6 that between cell 10 and 20 a "switch" from the fourth order mode structure to an envelope parametric instability occurs. It is accompanied by a clear two-fold

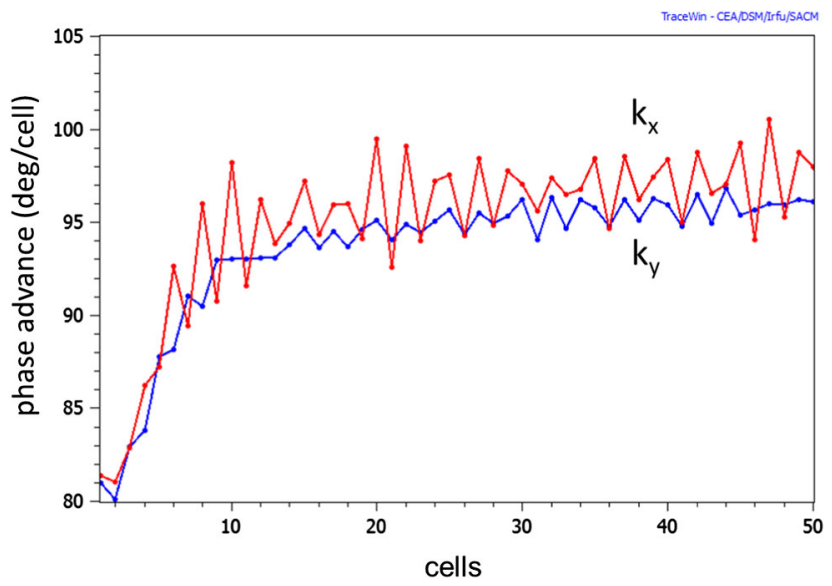

FIG. 5. rms phase advances versus cell number for waterbag example of Fig. 4.

symmetry in $x-x^{\prime}$-as recently studied in more detail in Ref. [9]. This phenomenon is also supported well by a density plot along the channel as shown in Fig. 7, where the bunch core density is graphically enhanced by contour lines. The envelope activity between cells 10 and 20 is accompanied by a strong coherent evolution, where the motion no longer follows the initial matched beam
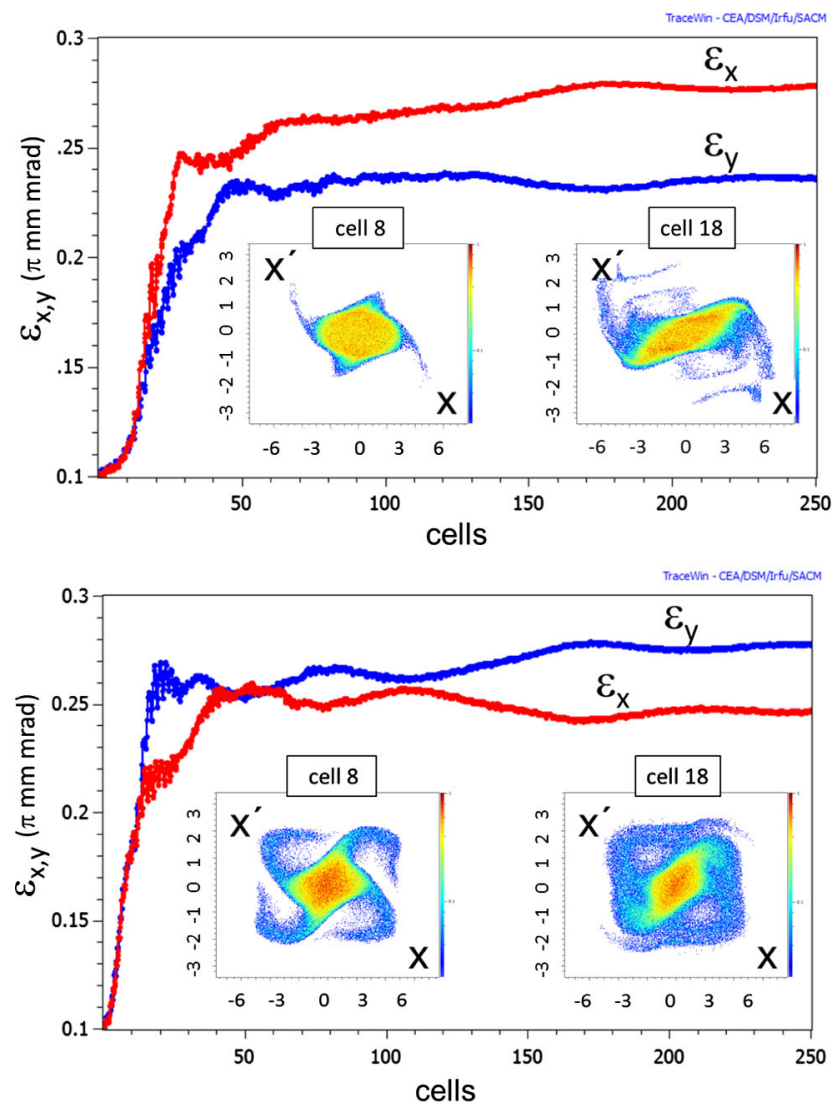

FIG. 6. rms emittances with phase space inserts for $k_{0, x, y}=120^{\circ}, k_{x, y}=73^{\circ}$ for $\mathrm{KV}$ beam (top graph) and waterbag distribution beam (bottom graph). 


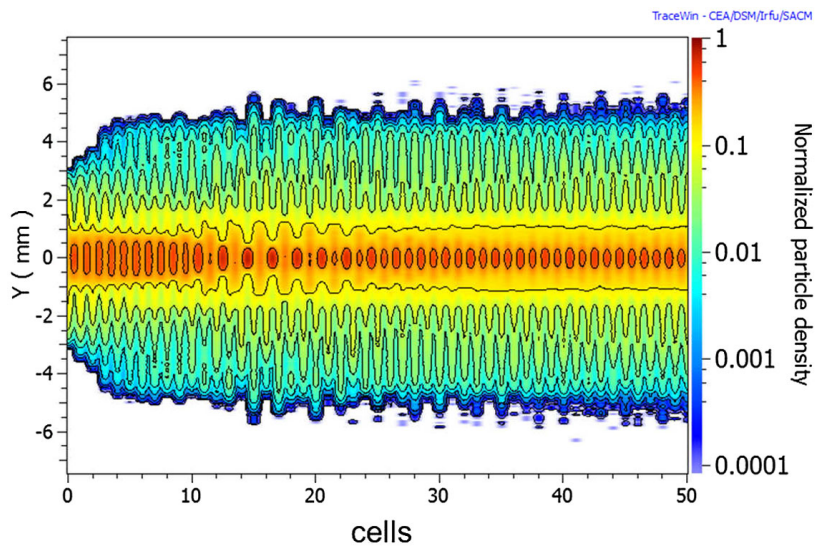

FIG. 7. Density in $y$ with contour plots for $k_{0, x, y}=120^{\circ}, k_{x, y}=$ $73^{\circ}$ for waterbag distribution beam.

contours, but 2 cells are needed to perform one period of the mode, which reflects the $180^{\circ}$ (or $n=1$ type) parametric resonance. Between cells 20-25 the coherent pattern gradually turns again into the incoherent pattern of the matched beam. The difference in behavior between the weaker and higher space charge cases can be understood in terms of collective detuning. As shown in Fig. 5 the weaker space charge case of Fig. 4 indicates that within less than 10 cells the fourth order single particle resonance has shifted the rms phase advance above $85^{\circ}$; by using the KVenvelope solver of TRACEWIN we find that this value is exactly the upper edge of the stopband of the envelope instability for $k_{0, x, y}=120^{\circ}$. With higher intensity, instead, the initial tune is lower and the - then faster growingenvelope mode has more time to develop before $k_{x, y}$ reaches the $85^{\circ}$ edge.

We emphasize here that we cannot confirm the assertion of Ref. [11] claiming that the envelope mode is part of the fourth order mode. As a mechanism, the envelope instability must be seen fully independent of the fourth order single particle resonance: first due to its nature as half-integer parametric resonance, second due to the fact that it follows directly from the envelope equations, which have no fourth order ingredients. This is not changed by the fact that a preceding fourth order resonance may cause a shift of tunes outside the envelope stopband-as described above.

Note that the splitting of transverse emittances beyond a certain cell in Fig. 4 is not explained in this context and is possibly a parametric emittance exchange mode. We find that the behaviour changes for larger space charge shift, like in the example of Fig. 7. We also note that an initial mismatch can induce an earlier appearance of the envelope instability and even make it the dominant phenomenon [9].

\section{SUM PARAMETRIC RESONANCES}

The occurrence of the envelope instability is not limited to a stopband near $90^{\circ}$, if at least two degrees of freedom are involved, where one tune is above, the other one below $90^{\circ}$ and sufficient coupling - here by space charge-between the two degrees of freedom exists as was recently shown in Ref. [12]. According to this work the space charge term in the coupled envelope equations, alternatively an infinitesimally small skew motion of the beam, naturally provide such a coupling. This may lead to a "sum parametric resonance," for which two different modes exist: (i) a coupled (even) mode sum instability involving two envelope modes and observing the parametric resonance condition $\phi_{1}+\phi_{2}=360^{\circ}$, and (ii) a single odd (also called "skew") second order mode (of the kind described in Ref. [16]) is driven unstable under the condition $\phi_{\text {skew }}=180^{\circ}$. Following Ref. [12], both cases result in a half-integer sum resonance condition of the form

$$
k_{0, x}+k_{0, y}-\Delta k_{s, \mathrm{coh}}=180^{0},
$$

where $\Delta k_{s, \text { coh }}$ is again a space charge dependent coherent shift specific to the particular mode. In smooth approximation it is found to be $\frac{5}{4} \Delta k_{\text {inc }}$ in the first case, and $\frac{7}{4} \Delta k_{\text {inc }}$ in the second case, with $\Delta k_{\text {inc }}(>0)$ the incoherent space charge tune shift. Equation (4) causes instability, if one tune is above $90^{\circ}$ and the other one suitably below, while satisfying the sum rule of Eq. (4).
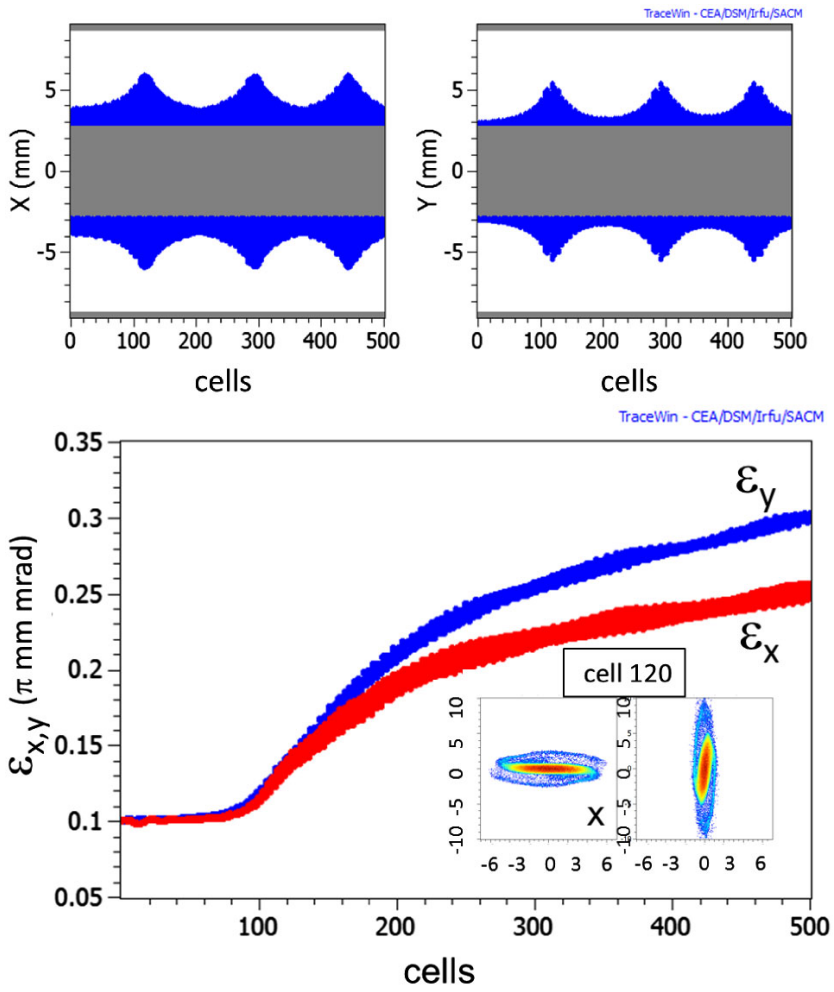

FIG. 8. Top graph: KV-envelopes versus cell number for $k_{0, x}=60^{\circ}, k_{0, y}=140^{\circ}$; bottom graph: simulation results for rms emittances versus cell number for waterbag distribution, with phase space inserts at cell 120 . 


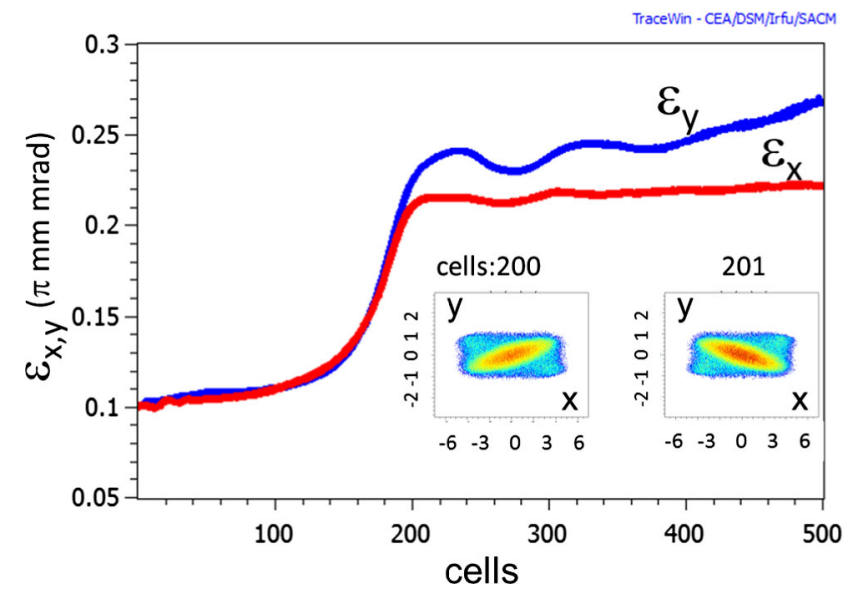

FIG. 9. rms emittances versus cell number for $k_{0, x}=60^{\circ}$, $k_{0, y}=145^{\circ}$ for waterbag distribution, with $x-y$ real space inserts at consecutive cells 200 and 201 .

An example of the coupled mode sum instability for significantly split tunes of $k_{0, x}=60^{\circ}, k_{0, y}=140^{\circ}$, and $\Delta k=19.5^{\circ}$-well satisfying Eq. (4)—is presented in Fig. 8 (with $k_{0, z}=50^{\circ}$ ). The KV-envelope shows a highly periodic behavior of exponential growth and nonlinear detuning. Note the difference with Fig. 1, where two independently oscillating, but weakly coupled envelopes produce a chaotic pattern in time. The corresponding full simulation shows a significant rms emittance growth, while the phase space inserts at cell 120 confirm the second order nature of the coupled envelope mode.

As suggested by the 2D findings of Ref. [12], increasing $k_{0, y}$ by 5 degrees to $145^{\circ}$ excites the adjacent single odd mode sum instability in our example, which is shown in Fig. 9. The characteristic skewing of the $x-y$ projection requiring two consecutive cells for one skewing period confirms the half-integer $180^{\circ}$ condition. Whether or not other types of coupled mode parametric resonances exist is still open and needs further research.

This sum parametric resonance differs substantially from the well-known linear coupling sum resonances, which require an external skew magnet component and replacement of the $180^{\circ}$ condition by $360^{\circ}$-besides replacing the coherent shift by the incoherent one.

\section{HIGHER ORDER PARAMETRIC RESONANCES}

Beyond the cases discussed in Sec. III, other parametric resonances have received less attention in high intensity beam dynamics. This might be partly due to their weaker effects, if compared with the $90^{\circ}$ stopband phenomena. For strong space charge effects as in high current linacs, however, their effect may be non-negligible, if the respective resonance conditions are satisfied over a sufficient large number of cells.

\section{A. Third order instability}

A third order parametric instability with $3 k_{0, x, y}-$ $\Delta k_{3, \text { coh }}=180^{\circ}$ is predicted in Fig. 3 for the $60^{\circ}$ stopband. This particular mode was believed to be the major current limitation in the early work on heavy ion inertial fusion, after it was realized that an upper limit of phase advance was given by the $90^{\circ}$ envelope instability stopband [1]. Note that a beam symmetric in $x$ and $y$ has no space charge multipole to excite a third order resonance phenomenon, except by driving it from initial noise via a parametric instability.

The third order mode is retrieved by our 3D simulations for the example $k_{0, x, y}=90^{\circ}$, if $k_{x, y}$ is varied over the range predicted in Fig. 3. For a waterbag distribution and $k_{x, y}=41.5^{\circ}$-close to the maximum tune shift in this stopband - the result is shown in Fig. 10. The growth of the rms emittance is preceded by a weak sixth order single particle (incoherent) resonance $6 k_{x, y}=360^{\circ}$ as indicated
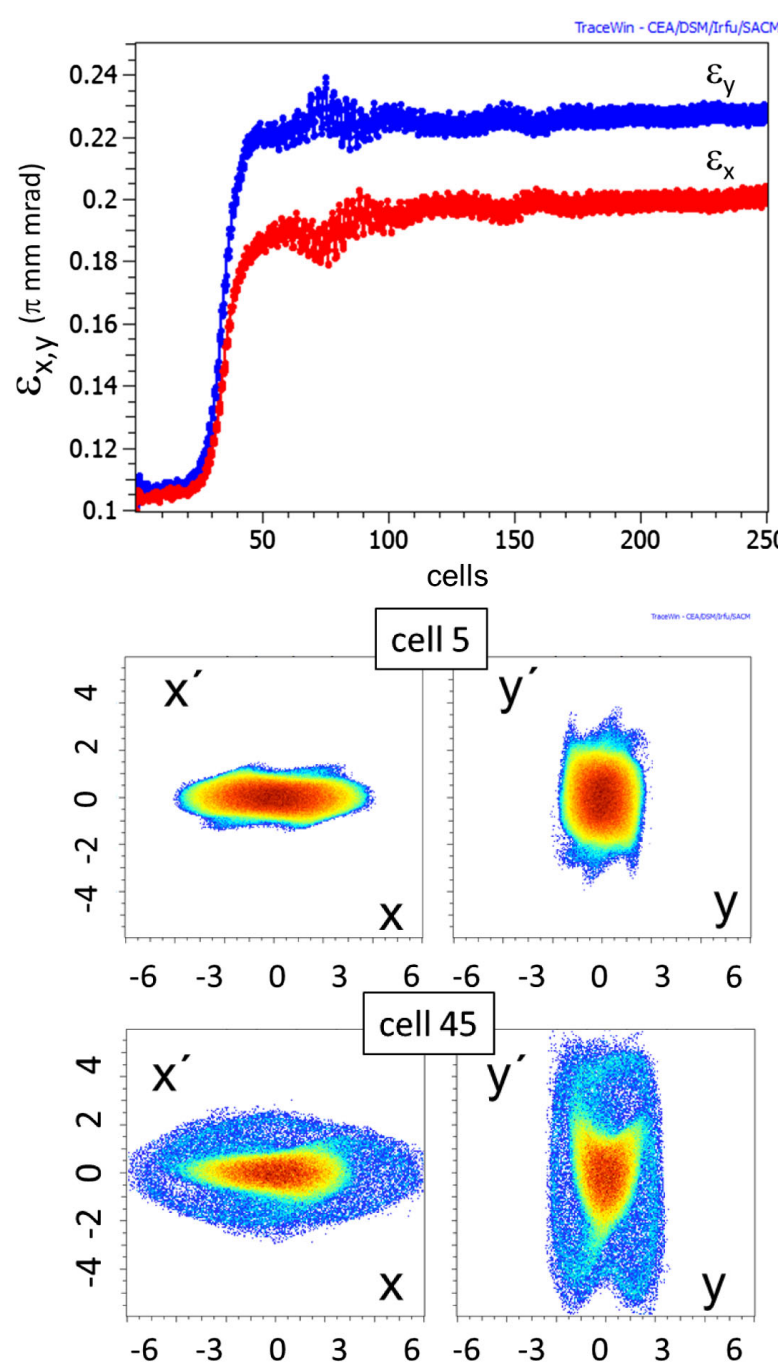

FIG. 10. Top graph: rms emittances versus cell number for $k_{0, x, y}=90^{\circ}, \quad k_{x, y}=41.5^{\circ}$ for waterbag distribution. Bottom graph: phase space plots at cells 5 and 45 . 
by the phase space symmetry at cell 5 . This phenomenon is analogous to that encountered in the interplay of fourth order and second order as discussed in Sec. III. It is, however, less pronounced here-possibly due to a weaker effect of the sixth order term.

The main growth of rms emittance is accompanied by a three-fold phase space structure as shown on the cell 45 phase space plot. The "triangles" repeat their orientation every second lattice cell, which confirms the $180^{\circ}$ condition, where the pseudo-sextupole is driven parametrically from noise until saturation. It results in more than doubling of the rms emittances in this example. The highest level of total growth is obtained for the case of maximum space charge tune depression, which is given at the lower edge of the stopband. While the emittance rises steeply in the exponential phase of the instability, it levels off following cell 50 .

We find that the rms phase advance self-consistently evolves close to $k_{x, y}=60^{\circ}$, which is the upper (i.e., low space charge density) edge of the stop-band calculated in Fig. 3. This agreement between waterbag simulation and linearized 2D KV-Vlasov theory is remarkable-as mentioned before in Sec. III. The question must be raised here, why the fast modulation of the space charge dependent $k_{x, y}$ of individual particles during a longitudinal period does not suppress the parametric instability effect.

\section{B. Fourth order and beyond}

In Sec. III we have already discussed the integer case of the fourth order parametric resonance $4 k_{0, x, y}-$ $\Delta k_{4, \mathrm{coh}}=360^{\circ}$, which was found for the KV distribution. For the initial waterbag distribution, instead, the picture was dominated by the single particle fourth order resonance $4 k_{x, y}=360^{\circ}$ driven by the initial space charge pseudooctupole.

The existence of a half-integer parametric instability $4 k_{0, x, y}-\Delta k_{4, \mathrm{coh}}=180^{\circ}$ (noting that this $\Delta k_{4, \mathrm{coh}}$ may be different from the integer case) is predicted in the bottom graph of Fig. 3, with a stopband near $45^{\circ}$. For a waterbag distribution we choose $k_{0, x, y}=70^{\circ}$ and $k_{x, y}=35^{\circ}$. The latter value can be assumed to be the lower stopband edge, if we extrapolate from the curves for $60^{\circ}$ and $80^{\circ}$ in Fig. 3 . Using $k_{0, z}=50^{\circ}\left(k_{z}=17^{\circ}\right)$ as in the examples before, we find a weak evidence of this mode, with only $4 \%$ emittance growth. However, by raising $k_{0, z}$ to $120^{\circ}$ we obtain a $30 \%$ emittance growth as is shown in Fig. 11, with the phase space insert at cell 20 confirming the fourth order structure. We assume that the roughly 5 times faster effective synchrotron oscillation plays a role and possibly reduces the transverse Landau damping effect, which needs additional study. Note that during the instability phase-similar to the parametric examples above- the effective tune rises from 35 to 41 degrees, which is consistent with the upper stopband ends in Fig. 3 (bottom graph) of the curves for $k_{0, x, y}$ of $60^{\circ}$ and $80^{\circ}$.

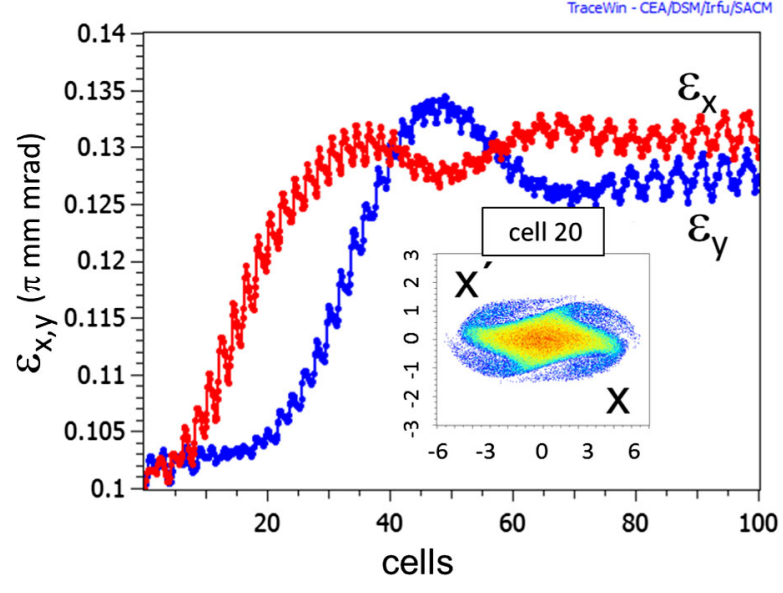

FIG. 11. rms emittances versus cell number for $k_{0, x, y}=70^{\circ}$, $k_{x, y}=35^{\circ}$ and waterbag distribution, with $x-x^{\prime}$ insert.

The half-integer nature eliminates the possibility of a simultaneously appearing single particle fourth order resonance as is found in the simulation of Sec. III for the $90^{\circ}$ stopband and waterbag distribution. Theoretically, one might expect an eighth order $8 k_{x, y}=360^{\circ}$ single particle resonance here, but no evidence for it is seen in the simulation.

Using KV-beams we find still higher than fourth order parametric cases in simulation-predicted by the analytical theory to appear in all orders-but with relatively small emittance effects. For corresponding waterbag beams no clear signatures of such higher order parametric modes could be seen. We thus conclude that for realistic beam distributions parametric resonances are likely to be insignificant beyond fourth order-at least in the range of parameters studied here.

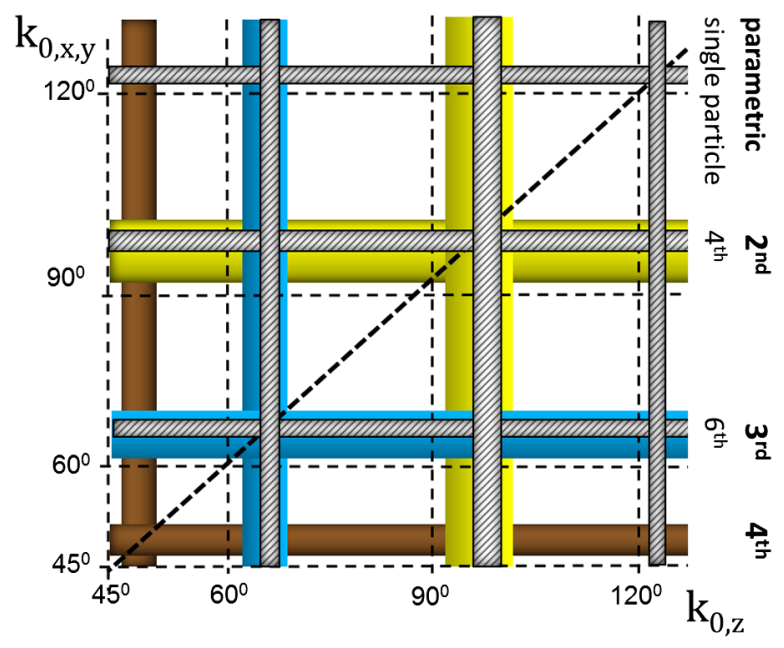

FIG. 12. Schematic stability diagram showing the location of transverse space charge driven 2nd to 4th order parametric resonances; and relevant 4th and 6th order single particle resonances (hatched, uncoloured bars). 


\section{SPACE CHARGE RESONANCE STABILITY DIAGRAM}

An overview of the above discussed transverse resonance phenomena as confirmed by our simulations of non$\mathrm{KV}$ distributions is summarized in the schematic "space charge resonance stability diagram" of Fig. 12-analogous to the resonance diagrams commonly used for circular machines. Analogous longitudinal resonances-not considered here-are equally marked for completeness. Note that the different order stopbands in Fig. 12 are indicated in the plane $k_{0, x, y}-k_{0, z}$, hence they are all shifted upwards by finite space charge as compared with the limits for small space charge. These shifts are only indicated schematically and not representative for the actual shifts resulting from theory or simulation. Besides the parametric stopbands of order 2-4, which are all of the half-integer type, the simultaneously found single-particle resonances of order 4 and 6 are also indicated (hatched, uncolored).

For completeness we have included the range above $120^{\circ}$. There, an integer type third order parametric resonance of the kind $3 k_{0, x, y}-\Delta k_{3, \text { coh }}=360^{\circ}$ might be expected theoretically. In simulations of waterbag or Gaussian beams no indication was found for it, which could be owed to the fact that integer parametric resonances are much weaker than the half integer cases of the same order and thus suppressed for non-KV beams. Instead, simulations show that single particle resonances of the kind $m k_{x, y}=360^{\circ} h$ can be excited by the matched beam space charge from higher harmonics of the lattice function, like a sixth order resonance with $m=6, h=2$ (indicated by the hatched bar at $120^{\circ}$ ), and even higher order as discussed in Ref. [20].

\section{CONCLUSIONS AND OUTLOOK}

This study shows that in high intensity beams a diversity of purely space charge driven parametric resonances exists - beyond the well-known envelope instability case. They are all characterized by the fact that the space charge driving term itself grows from noise or initial fluctuations due to the parametric process, and unstable modes grow exponentially until nonlinear saturation. Not insignificant levels of rms emittance growth are a result-at least in the cases up to fourth order. Parametric sum resonances with noticeable emittance growth effects have also been identified to occur between the transverse planes, for example, if tunes are suitably split.

For interpreting these low order instabilities in 3D as parametric resonances it is found that a comparison with previous analytical work for $2 \mathrm{D} \mathrm{KV}$-distributions provides excellent guidance as far as location and extent of stopbands is concerned. For realistic distributions we have retrieved, however, only the predicted half-integer $\left(180^{\circ}\right)$ cases, while integer cases appear to be suppressed and high order stopbands are completely absent. In at least two cases we find that these half-integer parametric resonances are accompanied by single particle resonances of twice the order. Stopbands are overlapping, which may result in competing effects.

In applications to very high-current linacs it may be generally safe to avoid all of the discussed stopbands up to fourth order even though linac lattices usually are not strictly periodic, with a limited number of cells and changing lattice structures as well as phase advances.

From a high intensity beam dynamics point of view, future work needs to address more systematically the phenomenon of parametric modes and reasons for their suppression. Factors that might play a role are the type of distribution function and the role of the longitudinal or synchrotron oscillation. It must be assumed that transverse Landau damping is not independent of the longitudinal motion. Effective Landau damping might contribute to the suppression of many of the theoretically predicted high order unstable modes of 2D KV distributions. Further work is needed to clarify, if fast synchrotron motion-as in our short bunches - can explain why effective Landau damping appears to be absent for the low order bunched beam parametric instabilities found in simulation.

[1] I. Hofmann, L. J. Laslett, L. Smith, and I. Haber, Stability of the Kapchinskij-Vladimirskij (K-V) distribution in long periodic transport systems, Part. Accel. 13, 145 (1983).

[2] J. Struckmeier and M. Reiser, Theoretical studies of envelope oscillations and instabilities of mismatched intense charged-particle beams in periodic focusing channels, Part. Accel. 14, 227 (1984).

[3] S. M. Lund and B. Bukh, Stability properties of the transverse envelope equations describing intense ion beam transport, Phys. Rev. ST Accel. Beams 7, 024801 (2004).

[4] C. Li and Y. L. Zhao, Envelope instability and the fourth order resonance, Phys. Rev. ST Accel. Beams 17, 124202 (2014).

[5] J. R. Pierce, Theory and Design of Electron Beams (D. Van Nostrand Co., New York, 1954).

[6] M. G. Tiefenback and D. Keefe, Measurements of stability limits for a space-charge-dominated ion beam in a long a. G. transport channel, IEEE Trans. Nucl. Sci. 32, 2483 (1985).

[7] M. G. Tiefenback, Ph.D thesis, U.C. Berkeley, 1986; Lawrence Berkeley Laboratory Report No. LBL-22465, 1986.

[8] L. Groening, W. Barth, W. Bayer, G. Clemente, L. Dahl, P. Forck, P. Gerhard, I. Hofmann, M. S. Kaiser, M. Maier, S. Mickat, T. Milosic, D. Jeon, and D. Uriot, Experimental Evidence of the $90^{\circ}$ Stop Band in the GSI UNILAC, Phys. Rev. Lett. 102, 234801 (2009).

[9] I. Hofmann and O. Boine-Frankenheim, Space-Charge Structural Instabilities and Resonances in High-Intensity Beams, Phys. Rev. Lett. 115, 204802 (2015).

[10] D. Jeon, Experimental evidence of space charge driven resonances in high intensity linear accelerators, Phys. Rev. Accel. Beams 19, 010101 (2016). 
[11] D. Jeon, J. H. Jang, and H. Jin, Interplay of spacecharge fourth order resonance and envelope instability, Nucl. Instrum. Methods Phys. Res., Sect. A 832, 43 (2016).

[12] O. Boine-Frankenheim, I. Hofmann, and J. Struckmeier, Parametric sum envelope instability of periodically focused intense beams, Phys. Plasmas 23, 090705 (2016).

[13] I. Hofmann and O. Boine-Frankenheim, Resonant Emittance Transfer Driven by Space Charge, Phys. Rev. Lett. 87, 034802 (2001).

[14] F. Verhulst, in Mathematics of Complexity and Dynamical Systems, edited by A. R. Meyers (Springer, New York, 2011), p. 1251.

[15] I. Hoveijn, The stability of parametrically forced coupled oscillators in sum resonance, Z. Angew. Math. Phys. 46, 384 (1995).
[16] I. Hofmann, Stability of anisotropic beams with space charge, Phys. Rev. E 57, 4713 (1998).

[17] D. Uriot and N. Pichoff, in Proceedings of IPAC2015, Richmond, VA, USA (JACoW, Virginia, 2015), paper MOPWA008.

[18] I. Hofmann, Influence of the distribution function on eigenoscillations and stability of a beam, Phys. Fluids 23, 296 (1980).

[19] I. Hofmann and O. Boine-Frankenheim, Grid dependent noise and entropy growth in anisotropic 3D particle-in-cell simulation of high intensity beams, Phys. Rev. ST Accel. Beams 17, 124201 (2014).

[20] I. Hofmann, in 57th ICFA beam dynamics workshop HB2016, Malmoe, Sweden, 2016 (JACoW, Malmoe, 2016), paper THPM1X01. 